\title{
DOOR DETECTION ALGORITHM OF MOBILE ROBOT IN HALLWAY USING PC-CAMERA
}

\author{
Jung-Suk Lee* Nakju Lett Doh* Wan Kyun Chung* Bum-Jae You** Young Il Youm* \\ *Robotics \& Bio-Mechatronics Lab., Mechanical Engineering, \\ Pohang University of Science \& Technology(POSTECH), Pohang, KOREA \\ E-mail:\{badol,nakji,wkchung,youm\}@postech.ac.kr \\ **Intelligent System Control Lab., \\ Korea Institute of Science and Technology(KIST), Seoul, KOREA \\ E-mail : ybj@kist.re.kr
}

\begin{abstract}
In this paper, an algorithm for door detection using a cheap PC-camera is developed based on the Bayesian network in a hallway where doors are located at various depth. The open/close status of the door is also important in developing a GVG-SLAM and we extracted five important door features from the image and distance data. We applied the Bayesian network algorithm and an entropy concept to increase the robustness. Experiment result shows that the proposed algorithm can help the GVG-SLAM of mobile robot by successfully identifying the status of the door.
\end{abstract}

Keywords: GVG- SLAM, door detection, low-cost sensor system, Bayesian-network

\section{INTRODUCTION}

The Generalized Voronoi Graph(GVG) [1, 2] is a good tool for the SLAM, especially in a hallway environment. A SLAM algorithm using the GVG (GVG-SLAM, in short) adds a node in a topologically meaningful place and localizes a robot at each node.

The weakness of the GVG-SLAM is that it cannot be used in a dynamic environment because there appears an unexpected node. In this case, the GVG-SLAM is apt to fail because it will localize the robot in a wrong place at the unexpected node.

One typical source of the unexpected node is the status and existence of the door in the hallway environment. So we need to detect door during the GVG-SLAM to mark that position as a place where an unexpected node could appear.

Previous door detection algorithms [3-7], however, cannot be used for the GVG-SLAM. The first reason is that previous results have assumed that the whole image of the door could be acquired. But a partial image of the door can be detected during the GVGSLAM because the GVG is a set of midpoints in the hallway and thus close to the door. The second reason is that previous algorithms are designed to detect doors given the map. So those cannot be directed ported to the SLAM.

We propose a door detection algorithm which uses the partial image of the door for the GVG-SLAM. To detect the door from the image, we extracted five important door features and applied Bayesian network algorithm. To increase the robustness of a door detection result, we adopted an entropy concept
[8] to represent the reliability of the result for each door.

This paper is organized as follows. In section 2, five features and the method to obtain these features are introduced. Section 3 suggests the algorithm for door detection. Experimental results of this algorithm are shown in Section 4. Finally, conclusion follows in Section 5 .

\section{FEATURES FOR DOOR DETECTION}

The door is a part of dynamic environment whose state can be changed but the wall is a static one that is fixed. In the case of the GVG-SLAM, the status of the door can disturb localization of a mobile robot. We need to know the existence of doors and the location of them. The door has many different features such as color, material, width, height, unevenness, doorknob, etc. as shown Fig. 1(a),(b).

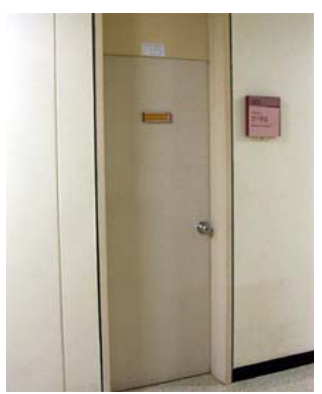

(a) The door

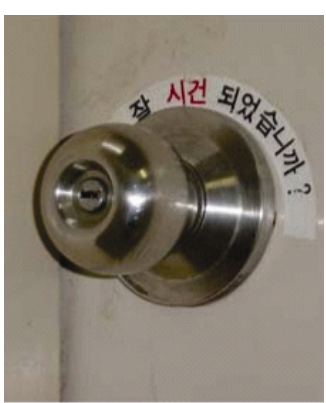

(b) The doorknob
Figure 1:Features of the door 


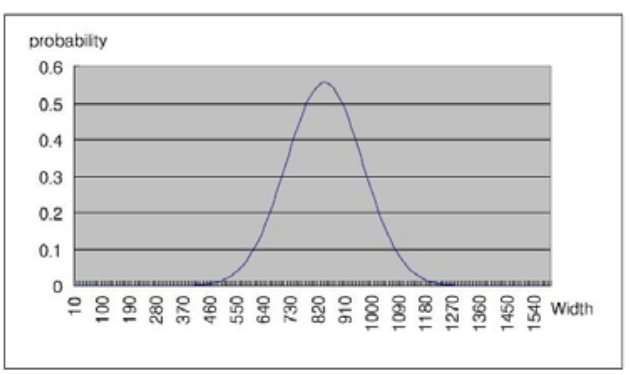

Figure 2:The Gaussian distribution for door width

Throughout this research, we have tried to make the sensor system as cheap as possible. It is important to reduce the sensor cost for commercial service mobile robots and we will not use laser range finder or experimental CCD camera for home service robot since those sensors raise the cost of the robot. A PCcamera that is connected to PC via USB(Universal Serial Bus) was selected to find vertical lines on the wall for dividing area and getting doorknob-like feature. The price of the PC-camera ranges from \$30 to $\$ 150$. Also we need a distance measure sensor to know depth change of the wall. For that purpose, we used inexpensive sonar sensor because our algorithm doesn't need high accuracy.

First of all, we divided the wall into a individual area by vertical lines. Each area has information about width, depth, doorknob. The width of area can be obtained from the distance of two adjacent vertical lines. The depth of area and change of open/close can be obtained from the distance measure sensor. The doorknob candidates can be obtained by the PCcamera. From these information we can aquire five features to determine the conditional possibility of the door as follows:

- The width of the area.

- The number of doorframes.

- The difference of depth.

- The existence of the doorknob.

- The state of door:[open/closed].

Now a detailed explanation of the above will be given. Firstly, the width of the area, whose value of the door in our experiment environment is assumed to be $790 \mathrm{~mm}$ or $890 \mathrm{~mm}$, can be used to distinguish between the door and the wall. To represent the probability of the door, we adopt the Gaussian distribution (Fig.2) whose peak $(\mu)$ is located at $840 \mathrm{~mm}$ and standard deviation $(\sigma)$ is 127.4 .

Secondly, the door has the doorframe at either or both sides. The doorframe is less than about $50 \mathrm{~mm}$ in our experiment environment. One-side-hinged door has two doorframe areas at both sides, a two-sidehinged door has only one doorframe area at either side(Fig.3). So the area that has the narrow area at both or either side has high probability than what doesn't have any narrow at either side.

Thirdly, the door is located deeper than the wall

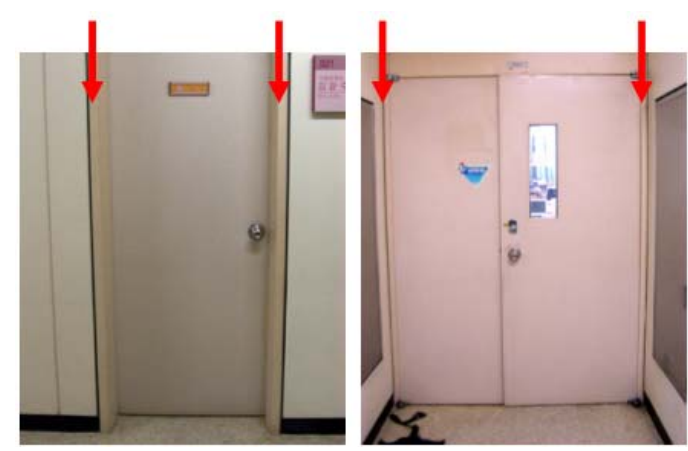

Figure 3: The number of doorframes

generally when the mobile robot sees them in the hallway but the depth is not fixed as shown in Fig.3. The difference of depth is another features for door detection.

Fourthly, the door normally has a doorknob. If we find a feature looks like doorknob, we assign high probability for the area in which includes the doorknob-like features.

Finally, the door can be opened or closed. Those states can be detected by the depth of the wall. If a depth of a wall is abruptly changed compare to a previous depth record, then we assign higher probability to be the door.

In the next section, we will explain a way to calculate the probability calculation.

\section{DOOR DETECTION ALGORITHM}

We calculate the conditional probability of the door by the Bayesian network [9-11]. The Bayesian network is a probabilistic graphical model that represent relation between variables of conditions. Nodes represent variables and arc lines represent dependencies between variables. Bayesian networks can represent causal and probabilistic processes easily. A node which induced current node is called a parent node. And the connected node which is hanged of from the parent node is called a child node. Using Bayesian network we make the graphical model that represents the probabilistic relations between conditions (Fig. 4). Each parent node (rectangle shape nodes in Fig. 4) represents five features. One child node(ellipse shape node in Fig. 4) represents the conditional probability whether the area is door or wall. Each parent condition is independent each other. The probability whether the area is door or wall can be calculated from parents node's conditional probability as follows:

$p(X \mid D W, D F, D, D K, O C)$

$=\frac{p(X, D W, D F, D, D K, O C)}{\sum_{X} p(X, D W, D F, D, D K, O C)}$

$=\frac{p(X, D W, D F, D, D K, O C)}{p(D W, D F, D, D K, O C)}$

$=p(X \mid D W) p(X \mid D W) p(X \mid D W) p(X \mid D W) p(X \mid D W)$ 


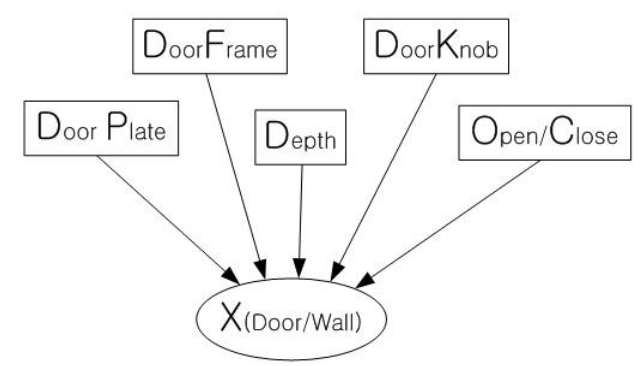

Figure 4: The Bayesian network for door detection

Table 1 : Variables

\begin{tabular}{|c|c|}
\hline Feature & Value \\
\hline$D W$ & Width (continuous value) \\
\hline$D F$ & $0,1,2$ \\
\hline$D$ & $-, 0,+$ \\
\hline$D K$ & Exist, not exist \\
\hline$O C$ & Change, not change \\
\hline
\end{tabular}

where $D W$ (door width), $D F$ (doorframe), $D$ (depth), $D K$ (doorknob) and $O C$ (open/close) denote the width of the area, the number of the doorframes, the difference of depth, the existences of the doorknob and the state of door, respectively. The variable $X$ corresponds to either a door or a wall. Each parent node has some values. Whose amounts are given as priories in Table 1.

The mobile robot starts the GVG-SLAM with the information of all in advance. Once the GVG-SLAM is started, the robot divided a acquired vision image into door/wall candidates by using vertical lines in the image. From the each candidates, five features $(D W / D F / D / D K / O C$ in Eq.(1)) are extracted and their corresponding probabilities area calculated. These possibilities area used to obtain the probability in Eq.(1).

We compare the value of probability for door and for wall. If the entropy $H$ (Eq.(2)) that measures the randomness or unpredictability is small, we determine the area is the door or the wall. But if the entropy is big, that means the result is not reliable. This area remains `the possible door area'.

$$
\begin{aligned}
& H=-p_{\text {door }} \log \left(p_{\text {door }}\right)-p_{\text {wall }} \log \left(p_{\text {wall }}\right) \\
& p_{\text {door }}=p\left(X_{\text {door }} \mid D W, D F, D, D K, O C\right) \\
& p_{\text {wall }}=p\left(X_{\text {wall }} \mid D W, D F, D, D K, O C\right)
\end{aligned}
$$

\section{EXPERIMENT}

\subsection{Experimental devices and sensors}

We applied our algorithm for the GVG-SLAM of the real mobile robot. The PARD(Postech Autonomous

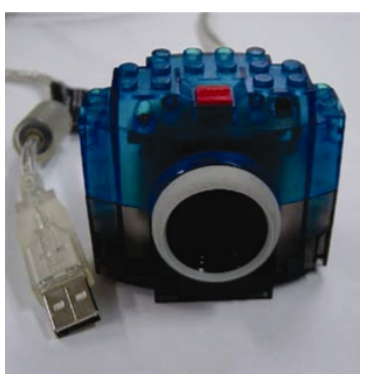

(a) Logitech Quickcam

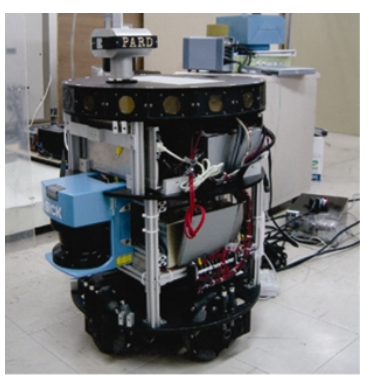

(b) PARD
Figure 5: Experimental device and sensor

Robotic Device) is used(Fig. 5(a)) as a testbed. This robot has omnidirectional wheel base, LRF(Laser Range Finder), gyroscope, sonar ring that consists of 16 sonar sensors and stereo vision system. But in this experiment, we used additional PC-camera and one directional data of LRF. The PC-camera is the Logitech Quickcam(Fig. 5(b))whose price is less than $\$ 100$. This camera is cheap and does not need additional device such as frame grabber. However, the image is noisy and has distortions. So accurate the image processing is not easy. Hence we only get the vertical line and door-like feature from image that is acquired from this camera. In our experiment, we also used the LRF to measure distances between the robot and the door/wall.

\subsection{Experimental environment and data}

The experimental environment is the third door of the fifth engineering building in POSTECH(Fig. 6). We had obtained the conditional probabilities of each feature before experiments. It is difficult to get enough data of this conditional probability because the variable of 'the width of the area' feature is continuous and width range is too wide. We made conditional probability for this feature as the Gaussian type probability distribution by referring 'Korea Standard door width`. The conditional probabilities of each feature are shown in Table 2 6. We tested our algorithm using these data.

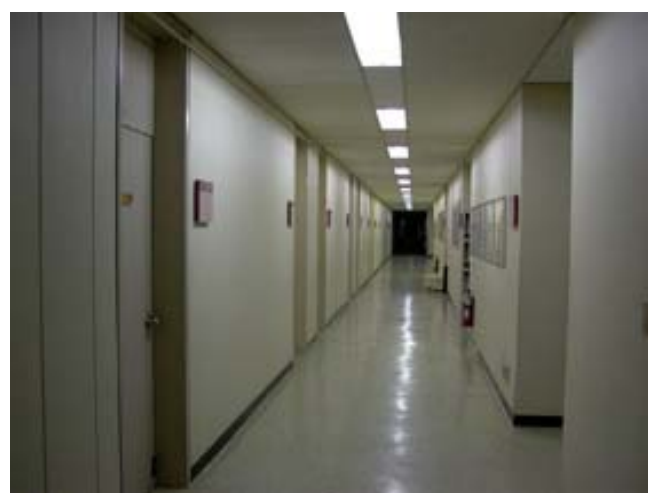

Figure 6: The Bayesian network for door detection 
Table 2: The width of the area

\begin{tabular}{|c|c|}
\hline DW & $\mathrm{X}=$ width of the area \\
\hline$p\left(X_{\text {door }} \mid D W\right)$ & $\frac{1}{1.8} \exp \left(-\frac{1}{2}\left(\frac{x-840}{127.4}\right)^{2}\right)$ \\
\hline$p\left(X_{\text {wall }} \mid D W\right)$ & $1-p\left(X_{\text {door }} \mid D W\right)$ \\
\hline
\end{tabular}

Table 3: The number of doorframe

\begin{tabular}{|c|c|c|c|}
\hline DF: & 2 & 1 & 0 \\
\hline$p\left(X_{\text {door }} \mid D F\right)$ & 0.6 & 0.5 & 0.4 \\
\hline$p\left(X_{\text {wall }} \mid D F\right)$ & 0.4 & 0.5 & 0.6 \\
\hline
\end{tabular}

Table 4: The difference of depth

\begin{tabular}{|c|c|c|c|}
\hline D: & + & 0 & - \\
\hline$p\left(X_{\text {door }} \mid D\right)$ & 0.5 & 0.04 & 0.91 \\
\hline$p\left(X_{\text {wall }} \mid D\right)$ & 0.5 & 0.96 & 0.09 \\
\hline
\end{tabular}

Table 5: The difference of depth

\begin{tabular}{|c|c|c|}
\hline DK: & Exist & Not exist \\
\hline$p\left(X_{\text {door }} \mid D K\right)$ & 0.6 & 0.03 \\
\hline$p\left(X_{\text {wall }} \mid D K\right)$ & 0.4 & 0.97 \\
\hline
\end{tabular}

Table 6: The state of door:[open/closed]

\begin{tabular}{|c|c|c|}
\hline OC: & change & No change \\
\hline$p\left(X_{\text {door }} \mid O C\right)$ & 0.94 & 0.5 \\
\hline$p\left(X_{\text {wall }} \mid O C\right)$ & 0.06 & 0.5 \\
\hline
\end{tabular}

\subsection{Experimental result}

In the hallway, there are four types of doors. Most ordinary door type is shown in Fig. 7(a). The door in Fig. 7(b) has some different features - no depth difference, only one doorframe. The door in Fig. 7(c),(d) has only one doorframe at one side and assist door. The assist door is narrower than common door. At first we close all doors and ran the robot to detect them. The result of first experiment is shown in Fig. 8. We omitted the data of the wall area because the conditional probability of those areas and entropy value are very small. So we can conffidently determine that these areas are the wall.

Secondly we opened some of the doors and did the same experiments. The result is shown in (Fig. 9). After an opening of 6th area, conditional probabilities of that area and the second area that has the same features with 6th are raised and the entropies are decreased. The algorithm determines those areas as the door area. We obtained the same result for the 15th and the 19th areas. In the cases of 11th and 12th areas, the conditional probability is increased but the entropy is increased after their opening. We call those area as 'a possible door area'. The 16th and 18th areas are rarely used. We have set these area closed. But we can decide that those areas are 'the possible door area' because those closed state

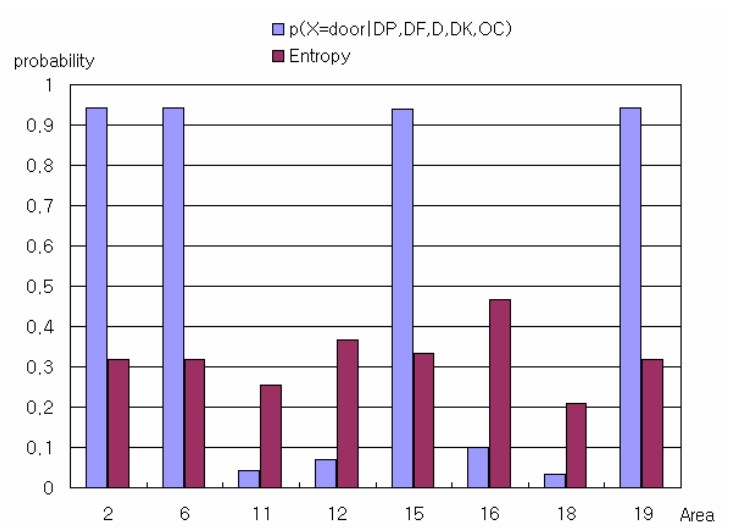

Figure 8: Experimental result before door open

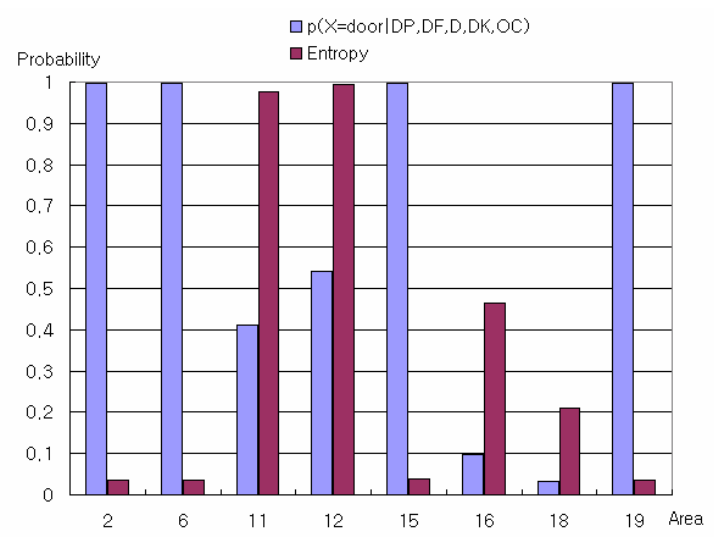

Figure 9: Experimental result after door open

area have many features similar to the real door. The mobile robot knows that this area could be opened in the future.

\section{CONCLUSION}

In this paper, we proposed a method to detect the door for the mobile robot during a GVG-SLAM by using a cheap PC-camera. The mobile robot acquires information of the wall with sensors. Then the robot selects five features that can match each area into either the wall or door. Bayesian Network is used to obtain the conditional probability whether the area is the door or the wall. The robot has the conditional probability of each feature before it makes a GVGmap. The probability value and the entropy determine whether the area is the door or the wall. Experiments show that this method can help the mobile robot make more complete GVG-map. Hence, the robot can estimate it's location more robustly.

Our method can be applied to a non-high performance system because this method uses probabilistic concept.

Since we have not conducted many experiments yet, we couldn't show robustness of our algorithm in other environment. We will verify how this algorithm 
can be used to aid the GVG-SLAM of mobile robot quantitatively. Lastly, we need more data of various environments to make the area 11,12,17 and 18 can be decided as a door.

\section{Acknowledgments}

This research was supported by the National Research Laboratory (NRL) Program (M1-0302-000040-03-J00-00-024-00) of the Ministry of Science \& Technology, Republic of Korea.

\section{REFERENCES}

[1] Howie Choset and Keiji Nagatani, “Topological Simultaneous Localization and Mapping(SLAM) : Toward Exact Localization Without Explicit Localization" , IEEE Trans. on Robotics and Automation, vol.17, no.2, 2001.

[2] Keiji Nagatani and Howie Choset, "Toward Robust Sensor Based Exploration by Constructing Reduced Generalized Voronoi Graph”, IEEE/RSJ Int. Conf. On Intelligent Robots and Systems, pp.1687-1692, 1999.

[3] T.Kanbara, J.Miura and Y,Shirai, "Selection of Effcient Landmarks for an Autonomous Vehicle" , IEEE/RSJ Int. Conf. on Intelligent Robots and Systems, vol.3, pp.1332-1338,1993.

[4] Hans Blaasvaer, Paolo Pirjanian and Henrik I. Christensen, "AMOR - An Autonomous Mobile Robot Navigation System”, IEEE Int. Conf. on Systems, Man, and Cybernetics, vol.3, pp.2266-2271, 1994.

[5] Dongsung Kim and Ramakant Nevatia, “A Method for Recognition and Localization of Generic Objects for Indoor Navigation" , IEEE Workshop on Application of Computer Vision, pp.280-288, 1994.

[6] Erhan Cokal and Abdulkadir Erden, "Development of an Image Processing System for a Special Purpose Mobile Robot Navigation" , IEEE Conf. on Mechatronics and Machine Vision in Practice, pp.246-252, 1997.

[7] Maurice R. Masliah and Robert W. Albrecht, "The Mobile Robot Surrogate Method for Developing Autonomy” , IEEE Trans. on Robotics and Automation, vol.14, no.2, pp.314-320, 1998.

[8] Richard O. Duda, Peter E. Hart and David G. Stork, Pattern Classiffcation, John Wiley \& Sons,Inc., 2001.
[9] Wray L. Buntine, “Operations for Learning with Graphical Models” , Journal of Artiffcial Intellgence Research, vol.2, pp.159-225, 1994.

[10] Michael I. Jordan, Learning in Graphical Models, The MIT press, 1999.

[11] Athanasios Papoulis and S. Unnikrishna Pillai, Probability, Random Variables and Stochastic Processes, McGraw-Hill,Inc., 2002.

[12] N. Doh, H. Choset and W. K. Chung, "Accurate Relative Localization Using Odometry” , IEEE Int. Conf. on Robotics and Automation, pp.1606-1612, 2003. 